\title{
The usefulness of antibodies to the BRCA1 protein in detecting the mutated BRCA1 gene. An immunohistochemical study
}

\author{
A Pérez-Vallés, M Martorell-Cebollada, E Nogueira-Vázquez, J A García-García, \\ E Fuster-Diana
}

\begin{abstract}
Aim-To assess the value of immunohistochemistry in discriminating between BRCA1 associated and non-BRCA1 associated breast tumours.

Methods-Four commercially available anti-BRCA1 antibodies were used on 45 paraffin wax embedded tumoral samples from patients with (seven of 45 ) and without (38 of 45) BRCA1 germline mutations. In all patients, the BRCA1 gene had been studied previously by means of the protein truncation test (PTT), conformational sensitive gel electrophoresis (CSGE), and direct sequencing of genomic DNA. Immunohistochemistry was carried out using the standard avidin-biotin immunoperoxidase method. Antigen retrieval was carried out by means of microwave pretreatment or autoclaving. The antibody panel used comprised D-20 (1/500), I-20 (1/100), K-18 (1/100), and MS110 (Ab-1; $1 / 50)$.
\end{abstract}

Results-No immunohistochemical differences in BRCA1 protein expression were found between cases with and without BRCA1 germline mutations. All positive cases showed predominantly cytoplasmic staining, in both tumoral and non-tumoral cells, with the polyclonal antibodies D-20, I-20, and K-18. After heating pretreatment both nuclear and cytoplasmic staining were found in tumoral and non-tumoral cells with the I-20 antibody. Only the monoclonal antibody MS110 showed a predominantly nuclear staining after microwave oven treatment. Conclusions-Commercially available BRCA1 antibodies lack the specificity required to identify the BRCA1 protein and thus are not useful for establishing differences between familial and sporadic breast tumours, or between BRCA1 associated and non-BRCA1 associated breast tumours.

(f Clin Pathol 2001;54:476-480)

Keywords: BRCA1; breast carcinoma; immunohistochemical study

BRCA1 is a putative tumour suppressor gene $^{12}$ linked to breast cancer in families with dominant inheritance of the disease. ${ }^{3}$ Many tumours with germline BRCA1 mutations display loss of heterozygosity $(\mathrm{LOH})^{4}$ at this locus, with loss of the wild-type BRCA1 allele, indicating a role for BRCA1 as a tumour suppressor gene.
BRCA1 is a large gene with a coding region of $5.5 \mathrm{~kb}$, expanded in 24 exons, and a total mRNA size of approximately $80 \mathrm{~kb}$, which is probably regulated by steroid hormones, oestrogen and progesterone. ${ }^{56}$ The protein has a RING finger domain at its $\mathrm{N}$-terminus ${ }^{3}$ and a BRCT motif at its C-terminus, ${ }^{7}$ which functions as a transactivator. ${ }^{89}$

The function of the BRCA1 protein is not fully elucidated but it probably plays an important role in DNA repair, apoptosis, or cell cycle arrest, interacting with p53 and RAD51 in response to DNA damage. ${ }^{10-13}$

There is much controversy with regard to the importance and localisation of BRCA1 protein expression. Chen et al reported that the BRCA1 protein is found in the nuclei of normal epithelial cells, but is aberrantly located in the cytoplasm of malignant mammary cells. $^{14}$ In contrast, Scully et al found the BRCA1 protein in the nuclei of both normal and malignant cells. ${ }^{15}$ Jensen et al reported that BRCA1 is located in the cytoplasm and the cell membrane, ${ }^{16}$ whereas Coene et al proposed that it is located in cytoplasmic tube-like invaginations in the nucleus. ${ }^{17}$ These contradictory results on the location of the BRCA1 protein could result from differences in the specificity of the antibodies used, the different fixation methods used, and the presence of splice variant isoforms of the BRCA1 protein in tumours.

Immunohistochemical studies on formalin fixed, paraffin wax embedded tumours have demonstrated a loss or reduction of protein expression not only in BRCA1 associated breast carcinomas but also in non-BRCA1 associated familial and sporadic breast carcinomas. ${ }^{18-20}$

Although the BRCA1 gene is not mutated in sporadic breast carcinomas, ${ }^{3}$ the loss or reduction of BRCA1 protein expression (as detected by immunohistochemistry $)^{20}$ and the high frequency of $\mathrm{LOH}^{21} 22$ indicate that the BRCA1 gene probably features in the genesis of sporadic breast cancer through a mechanism other than mutation.

In our study, we evaluate from a practical point of view the applicability and value of four different commercially available anti-BRCA1 antibodies (polyclonal D-20, I-20, and K-18 and monoclonal MS110) to discriminate between BRCA1 associated and non-BRCA1 associated tumours. We performed an immunohistochemical study on formalin fixed, paraffin wax embedded tumour samples from 45 patients with sporadic and inherited breast 
Table 1 Clinicopathological results

\begin{tabular}{|c|c|c|c|}
\hline & \multirow[b]{2}{*}{$\begin{array}{l}B R C A 1+ \\
(n=7)\end{array}$} & \multicolumn{2}{|c|}{$B R C A 1-(n=38)$} \\
\hline & & $\begin{array}{l}\text { Familial cases } \\
(n=16)\end{array}$ & $\begin{array}{l}\text { Sporadic cases } \\
(n=22)\end{array}$ \\
\hline \multicolumn{4}{|l|}{ Group at risk (family history) } \\
\hline High risk & 7 & 10 & \\
\hline Low risk & 0 & 6 & \\
\hline \multicolumn{4}{|l|}{ Age at diagnosis (years) } \\
\hline$\leqslant 45$ & 5 & 6 & 14 \\
\hline$>45$ & 2 & 10 & 8 \\
\hline \multicolumn{4}{|l|}{ Tumour size (cm) } \\
\hline $\mathrm{T} 1(\leqslant 2)$ & 3 & 8 & 8 \\
\hline T2 $(2-5)$ & 4 & 4 & 10 \\
\hline $\mathrm{T} 3(\geqslant 5)$ & 0 & 4 & 4 \\
\hline \multicolumn{4}{|l|}{ TNM stage } \\
\hline I & 2 & 8 & 4 \\
\hline II & 5 & 3 & 7 \\
\hline III & 0 & 5 & 11 \\
\hline \multicolumn{4}{|l|}{ Histological type (WHO) } \\
\hline Invasive ductal carcinoma & 3 & 12 & 19 \\
\hline Medullary carcinoma & 4 & 1 & 3 \\
\hline Invasive lobular carcinoma & 0 & 2 & 0 \\
\hline Mucinous carcinoma & 0 & 1 & 0 \\
\hline \multicolumn{4}{|c|}{ Histological grade (Bloom and Richardson, Nottingham) } \\
\hline Grade I & 0 & 7 & 7 \\
\hline Grade II & 2 & 7 & 12 \\
\hline Grade III & 5 & 2 & 3 \\
\hline \multicolumn{4}{|l|}{ Axillary lymph node status } \\
\hline Positive & 1 & 5 & 13 \\
\hline Negative & 6 & 11 & 9 \\
\hline \multicolumn{4}{|l|}{ Oestrogen receptor } \\
\hline Positive $(\geqslant 10 \mathrm{fmol} / \mathrm{mg}$ ) & 1 & 6 & 16 \\
\hline Negative (< 10 fmol/mg) & 6 & 10 & 6 \\
\hline \multicolumn{4}{|l|}{ Overall free survival (years) } \\
\hline$\leqslant 5$ & 4 & 9 & 14 \\
\hline$>5$ & 3 & 7 & 8 \\
\hline
\end{tabular}

Oestrogen receptor status was studied by enzyme immunoanalysis.

cancer, in whom germline mutations of the BRCA1 gene had been studied previously by means of the protein truncation test (PTT), conformational sensitive gel electrophoresis (CSGE), and direct sequencing of genomic DNA.

\section{Methods}

In total, 45 patients with both sporadic $(\mathrm{n}=22)$ and familial $(\mathrm{n}=23)$ breast cancer were selected. Familial cases had been stratified previously into high and low risk susceptibility groups according to the criteria of Hartmann et $a l .{ }^{23}$ Clinicopathological data were available in all cases (table 1). All tumours were histologically classified according to the WHO criteria ${ }^{24}$ and were graded according to the Nottingham modification ${ }^{25}$ of Bloom and Richardson.

A complete gene study in search of BRCA1 germline mutations had been carried out previously by means of PTT, CSGE, and direct sequencing of genomic DNA.
The antibody panel used comprised the polyclonal D-20, I-20, and K18 antibodies from Santa Cruz Biotechnologies (Santa Cruz, California, USA) and the monoclonal MS110 (Ab-1) antibody from Oncogene Research Products (Cambridge, Massachusetts, USA).

Immunohistochemistry was performed on formalin fixed, paraffin wax embedded samples using the standard avidin-biotin immunoperoxidase method. Two different methods were used for antigen retrieval: autoclaving in $0.01 \mathrm{M}$ sodium citrate at $121^{\circ} \mathrm{C}, 15$ pounds/ square inch for 30 minutes (for the polyclonal antibodies) and heating in a microwave oven at $500 \mathrm{~W}$ for 20 minutes in citrate buffer (for the monoclonal antibody). Slides were then incubated with the primary antibodies D-20 (1/500), I-20 (1/100), K18 (1/100), and MS110 (Ab-1; 1/50) and diluted with phosphate buffered saline (PBS) containing $0.5 \%$ Triton-X100 (vol/vol). Fixed, paraffin wax embedded myometrium served as a positive control, whereas similarly treated spleen tissue served as a negative control for the polyclonal antibodies, according to Jarvis and colleagues ${ }^{26}$; the Ab-1 antibody was probed in normal skin and the staining of the basal epithelial cells served as a positive control. The negative control in this case was obtained by omitting the antibody.

Tumours with cytoplasmic and/or nuclear staining were considered positive. The proportion of stained cells was scored as follows: 0 (no staining), + (less than $25 \%$ of tumoral cells stained), and ++ (more than $25 \%$ of tumoral cells stained).

\section{Results}

Table 1 shows the clinicopathological results. Briefly, tumours from patients with BRCA1 mutations were mostly medullar carcinomas, had a high histological grade, and were oestrogen receptor negative.

All patients with BRCA1 germline mutations were in the "familial" group and belonged to high risk families. Three different BRCA1 germline mutations were found, namely: 1623del5 (two patients), 3450del4 (four patients), and 185delAG (one patient). All resulted in truncated proteins.

Table 2 lists the mutations found and their coding effects.

Table 2 BRCA1 mutations and immunostaining

\begin{tabular}{|c|c|c|c|c|c|c|c|c|c|c|c|c|}
\hline \multirow[b]{3}{*}{ Sample } & \multirow[b]{3}{*}{ Mutation } & \multirow[b]{3}{*}{ Codon } & \multirow[b]{3}{*}{ Exon } & \multirow[b]{3}{*}{ Coding effect } & \multicolumn{8}{|c|}{ Immunostaining } \\
\hline & & & & & \multicolumn{2}{|c|}{$D-20$} & \multicolumn{2}{|c|}{$I-20$} & \multicolumn{2}{|c|}{$K-18$} & \multicolumn{2}{|c|}{ MS110 } \\
\hline & & & & & $N$ & $C$ & $N$ & C & $N$ & C & $N$ & C \\
\hline Case 1 & 1623 del5 & 541 & 11 & Frameshift & - & ++ & - & ++ & - & ++ & - & - \\
\hline Case 2 & 1623 del 5 & 541 & 11 & Frameshift & - & $++^{\star}$ & + & ++ & - & - & ++ & - \\
\hline Case 3 & $185 \mathrm{delAG}$ & 61 & 2 & Frameshift & - & ++ & + & ++ & - & ++ & - & - \\
\hline Case 4 & 3450del4 & 1150 & 11 & Frameshift & - & ++ & - & ++ & - & - & - & - \\
\hline Case 5 & 3450 del 4 & 1150 & 11 & Frameshift & - & ++ & + & ++ & - & - & + & - \\
\hline Case 6 & 3450del4 & 1150 & 11 & Frameshift & - & $++^{\star}$ & ++ & ++ & - & - & + & - \\
\hline Case 7 & 3450del4 & 1150 & 11 & Frameshift & - & ++ & ++ & ++ & - & + & ++ & - \\
\hline
\end{tabular}

Location of immunogen (amino acid): D-20, N -terminal (aa 2-21); I-20, C-terminal (aa 1826-46); K-18, N -terminal (aa 70-89); MS110, N -terminal (aa 1-304).

Antigen retrieval: D-20, I-20, and K-18, autoclaving; MS110, microwave.

Staining: C, cytoplasmic staining; $\mathrm{N}$, nuclear staining; ${ }^{\star}$ membrane pattern. 


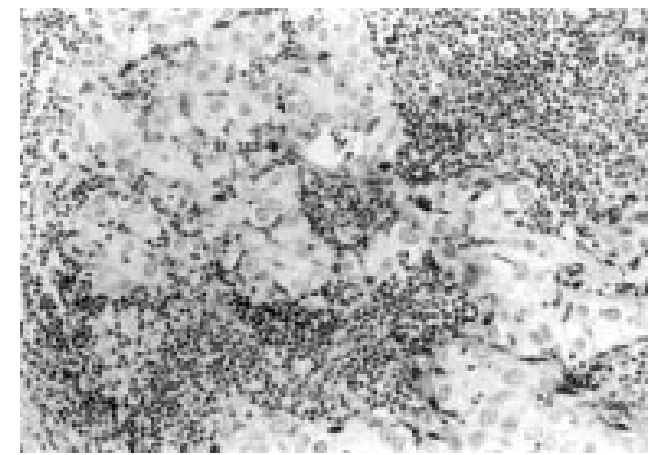

Figure $1 \quad A b-1$ negative staining in a familial breast cancer from a patient without BRCA1 mutations. Note the positive staining in stromal lymphocytes surrounding tumoral cells.

IMMUNOHISTOCHEMICAL STUDY

Staining was heterogeneous in intensity and localisation (cytoplasmic $v$ nuclear), mainly as a result of the antibody and the antigen retrieval method used.

After autoclaving, nuclear staining was negative in tumoral cells from patients with and without BRCA1 mutations with the D-20 and $\mathrm{K}-18$ polyclonal antibodies.

Twenty cases failed to show nuclear staining with the I-20 polyclonal antibody: 18 were from patients without BRCA1 mutations and corresponded to five familial and 13 sporadic breast tumours; the other two (cases 1 and 4) were from patients with BRCA1 germline mutations 1623del5 and 3450del4. Four tumours from patients carrying the same mutations (case 2 and cases 5, 6, and 7) showed intense nuclear staining with this same antibody.

After microwave pretreatment, 24 tumours did not show nuclear staining with the MS110 monoclonal antibody (fig 1); 21 were from patients without BRCA1 germline mutations and corresponded to 10 familial and 11 sporadic breast tumours; the other three (cases 1, 3, and 4) were from patients with BRCA1 germline mutations 1623del5, 185delAG, and $3450 d e 14$. The remaining four tumours, however, from patients with the $1623 \mathrm{del} 5$ (case 2)

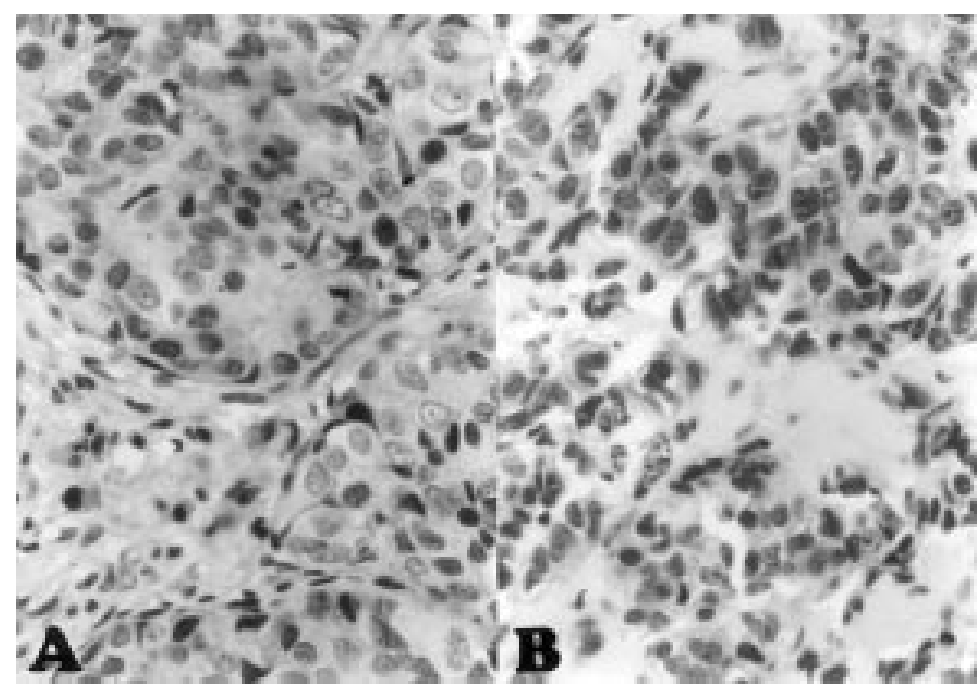

Figure $2 A b-1$ nuclear staining in familial breast cancer cases from patients $(A)$ with and (B) without BRCA1 germline mutations.

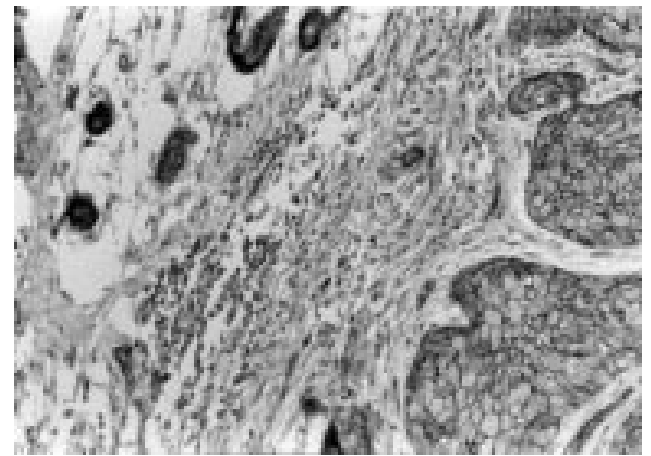

Figure 3 Membrane staining pattern with the D-20 antibody in a familial breast cancer from a patient with BRCA1 mutation.

and 3450del4 (cases 5, 6, and 7) mutations, showed nuclear staining; hence, the presence or absence of nuclear staining was not related to the presence or absence of BRCA1 germline mutations (fig 2), or to the type of BRCA1 mutation present.

Cytoplasmic staining was positive in all 45 cases with the D-20 and I-20 polyclonal antibodies, in contrast to the staining pattern found with the MS110 monoclonal antibody, which was predominantly nuclear, with the exception of one sample (case 24) without BRCA1 mutations. The K-18 polyclonal antibody gave positive results for cytoplasmic staining in 10 patients $(1,3$, and 7 all of whom had BRCA1 mutations). As with the nuclear staining, no correlation was found between staining and the presence or absence of BRCA1 germline mutations.

The cytoplasmic staining pattern was predominantly diffuse. A membrane staining pattern was also seen in 19 patients (patients 2 and 6 had BRCA1 mutations) when the D-20 antibody was used (fig 3); in addition, one patient (case 11) showed a granular cytoplasmic staining with the K-18 antibody (data not shown).

Results in non-tumoral cells present within the samples were similar to those observed in tumoral cells. No nuclear staining was seen with the D-20 and K-18 antibodies and only cytoplasmic staining was seen in these cases. We found cytoplasmic and nuclear staining in seven patients with the I-20 antibody, and two of these patients (cases 1 and 5) had BRCA1 germline mutations. Finally, with the MS110 antibody, non-tumoral cells showed a predominantly nuclear staining pattern in all patients (data not shown).

Tables 2 and 3 show the results of the immunohistochemical study. We found no correlation between BRCA1 staining and other prognostic factors, such as oestrogen receptor or axillary lymph node status. Only tumoral size (tumours $\geqslant 4 \mathrm{~cm}$ ) and certain histological types (medullar carcinoma) appeared to correlate with the absence of BRCA1 staining, whereas most of the grade I tumours were positive.

\section{Discussion}

In our study, we describe the use of immunohistochemistry on routinely processed, neutral formalin fixed and paraffin wax embedded 
Table 3 BRCA1 immunostaining in BRCA1 negative cases

\begin{tabular}{|c|c|c|c|c|c|c|c|c|}
\hline \multirow[b]{3}{*}{ Antibody } & \multicolumn{4}{|c|}{ Familial cases $(n=16)$} & \multicolumn{4}{|c|}{ Sporadic cases $(n=22)$} \\
\hline & \multicolumn{2}{|c|}{ Nuclear staining } & \multicolumn{2}{|c|}{ Cytoplasmic staining } & \multicolumn{2}{|c|}{ Nuclear staining } & \multicolumn{2}{|c|}{ Cytoplasmic staining } \\
\hline & Positive & Negative & Positive & Negative & Positive & Negative & Positive & Negative \\
\hline D-20 & 16 & 16 & - & - & 22 & 22 & - & - \\
\hline $\mathrm{I}-20$ & 11 & 5 & 15 & 1 & 9 & 13 & 22 & - \\
\hline $\mathrm{K}-18$ & 16 & 5 & 11 & - & 22 & 2 & 20 & - \\
\hline $\mathrm{Ab}-1$ & 6 & 10 & - & 16 & 11 & 11 & 1 & 21 \\
\hline
\end{tabular}

tumours from 45 patients with breast carcinoma (23 familial, 22 sporadic).

The BRCA1 gene had been studied previously in all cases, and three different BRCA1 germline mutations (185delAG, 1623del5, and 3450 del4) were found in seven patients from the familial group; all were frameshift mutations resulting in truncated proteins.

There is controversy regarding the size and localisation of the BRCA1 protein, mainly as a result of differences in fixation, ${ }^{15}{ }^{17}$ the antigen retrieval method used, and the specificity of anti-BRCA1 antibodies. ${ }^{27}{ }^{28}$ Using autoclaving and microwave antigen retrieval methods, we found that of all of the antibodies tested (D-20, I-20, K-18, and MS110), only MS110 (Ab-1) produced consistent results on routinely processed material.

Using the D-20 and K-18 antibodies, we found cytoplasmic staining only, the nucleus being constantly non-immunoreactive either in cancer or normal mammary gland cells in all samples tested. This agrees with data from a previous report ${ }^{29}$ in which only cytoplasmic staining was seen using polyclonal antibodies D-20, I-20, K-18, and C-20 on paraffin wax embedded samples. Our results differ from those of Jarvis, ${ }^{26}$ who found differences in nuclear staining of tumour cells with the D-20 antibody, the cytoplasm being positive in all sporadic breast cancers tested.

Using the C-terminal I-20 polyclonal antibody, we found no differences in staining between tumoral and non-tumoral mammary epithelial cells. Uniform cytoplasmic staining was seen in all samples with heterogeneous nuclear staining. Data have been published demonstrating I-20 false positive immunostaining using cell lines lacking the C-terminal portion of the BRCA1 protein ${ }^{19}$; furthermore, the I-20 antibody probably recognises proteins other than BRCA $1,{ }^{30}$ or products of alternative splicing of the BRCA1 gene. ${ }^{18}$

Although these polyclonal antibodies (D-20, I-20, and K-18) were valid for immunoprecipitation studies, their value for immunohistochemistry on frozen or paraffin wax embedded samples has been questioned. ${ }^{18}$

In our study, the monoclonal antibody MS110 (Ab-1) ${ }^{15}$ appeared to give the most accurate, reliable, and reproducible results when used on formalin fixed, paraffin wax embedded material. ${ }^{19} 20$

The MS110 antibody reacts with the $\mathrm{N}$-terminal portion of the BRCA1 protein only after antigen retrieval by heating (microwave or autoclaving), producing an almost exclusively nuclear staining pattern in normal mammary cells. ${ }^{15} 20$
Using the MS110 antibody and antigen retrieval with a microwave oven, we observed complete loss of nuclear staining in three of the seven familial BRCA1 associated breast cancers. Similar results were seen by Yoshikawa et $a l{ }^{19}$ who found a loss of nuclear signal in seven of the 19 BRCA1 associated breast carcinomas studied.

As expected, no signal was detected in the malignant cells in the only tumour containing the BRCA1 185delAG mutation, which results in the deletion of most of the protein, including the Ab-1 epitope. No nuclear signal was seen in tumour cells from patients carrying the 1623del5 and 3450del4 BRCA1 germline mutations, in agreement with previous reports in which the expression of BRCA1 mRNA in BRCA1 associated cancer was found to be low, regardless of the type of mutation, ${ }^{19}{ }^{31}$ because loss of the BRCA1 protein occurs irrespective of the position of the mutation in the BRCA1 gene. ${ }^{20}$

Some data from previous studies have shown that the BRCA1 protein is present in the cytoplasm. Splice variant isoforms that lack exon 11 along with the nuclear localisation signal are located exclusively in the cytoplasm. ${ }^{29}{ }^{32}$ However, we found no cytoplasmic staining with the MS110 antibody in the 45 breast cancers that we tested.

We found that different patients were carriers of the same truncating mutation: 1623del5 (patients 1 and 2) and 3450del4 (patients 4-7). Of particular interest was the fact that with the MS110 antibody negative nuclear staining was seen in patients 1 and 4 only, and staining was positive in the remainder of the tumours. The expression of BRCA1 mRNA is directly related to the number of wild-type alleles. ${ }^{33}$ The relatively high amounts of BRCA1 protein that we detected immunohistochemically in some of the tumours with truncated BRCA1 proteins, and the discrepancies between tumours carrying the same mutation, may result either from the retention of the wild-type allele or heterozygosity among cancer cells. ${ }^{19}{ }^{33}$ Results reported by Yoshikawa and colleagues ${ }^{19}$ support the idea that MS-110 could be useful in prescreening tumours for BRCA1 mutations, mainly because of the high detection rate (seven of nineteen) of alterations in the BRCA1 gene product in BRCA1 associated breast cancers. Our results (three of seven) are quite similar to those of Yoshikawa $^{19}$ when only the familial BRCA1 associated breast cancers are compared. Applying these criteria, our apparent detection rate of BRCA1 mutations using the MS110 antibody is highest in familial $(62.5 \%)$ and sporadic $(50 \%)$ non-BRCA1 associated breast carcinomas. The 
presence or absence of BRCA1 germline mutations was objectively demonstrated in all patients after the study of the BRCA1 gene by PTT, CSGE, and direct sequencing of genomic DNA.

Taylor et al reported the absence of nuclear and cytoplasmic BRCA1 expression in 142 non-familial breast cancers studied by immunohistochemistry, and loss of nuclear BRCA1 expression in $19 \%$ of the cases, indicating that BRCA1 plays a role in sporadic cancers. ${ }^{20}$ Similar results have been reported in other immunohistochemical studies on sporadic, non-inherited breast cancer that used the MS110 (Ab-1) antibody, with a reduction in nuclear BRCA1 expression in $27 \%,{ }^{19} 20 \%,{ }^{34}$ and $43.5 \%{ }^{18}$ of the tumours tested.

A reduction of BRCA 1 protein expression in sporadic carcinomas has been associated with high histological grade tumours, ${ }^{18}$ overexpres$\operatorname{sion}^{19}$ and loss $^{34}$ of the c-erb-B-2 protein, and high proliferative phenotype, perhaps related to truncations at the $\mathrm{N}$-terminal and $\mathrm{C}$-terminal portions of the BRCA1 protein. ${ }^{26}$ We did not find a relation between the loss of nuclear staining and histological grade, tumour type, other clinicopathological data, or expression of oestrogen or progesterone receptors, p53, cathepsin D, c-erbB-2, and bcl-2 using immunohistochemistry (data not shown).

These data are consistent with the observation that BRCA1 mRNA values are decreased in sporadic breast cancers ${ }^{35}$ and in breast cancer cell lines such as MCF-7. ${ }^{33}$ Concentrations of the BRCA1 protein are also decreased in mammary tumours compared with matched normal breast tissue, ${ }^{36}$ implying multiple mechanisms of BRCA1 expression downregulation in these tumours. Other data suggest that the reduced expression of the BRCA 1 protein might play an important role in mammary carcinogenesis in sporadic cancer, and that mechanisms other than mutation, ${ }^{19}{ }^{21}$ in particular $\mathrm{LOH},{ }^{29}$ might be involved in the reduced expression of the BRCA1 protein. Taken together, our data support the notion that the $\mathrm{N}$-terminal specific MS110 antibody is not useful in prescreening BRCA1 mutations because it has a lower rate of detection in breast tumours from patients carrying a BRCA1 gene mutation than in tumours, both familial $(65 \%)$ or sporadic $(50 \%)$, from patients without BRCA1 germline mutations. This fact, together with the inconsistency in immunohistochemical results among patients carrying the same truncating BRCA1 mutation, points to the non-specificity of the MS1 10 antibody in the prescreening of BRCA1 associated mammary cancers.

1 Holt JT, Thompson ME, Szabo C, et al. Growth retardation and tumor inhibition by BRCA1. Nat Genet 1996;12:298302 .

2 Rao VN, Shao N, Ahmad M, et al. Antisense RNA to the putative tumor suppressor gene BRCA1 transforms mouse fibroblasts. Oncogene 1996;12:523-8.

3 Miki Y, Swensen J, Shattuck-Eidens D, et al. A strong candidate for the breast and ovarian cancer susceptibility gene BRCA1. Science 1994;266:66-71.

4 Smith SA, Easton DF, Evans DGR, et al. Allele losses in the region $17 \mathrm{q} 12-21$ in familial breast and ovarian cancer involve the wild-type chromosome. Nat Genet 1992;2:12831 .

Gudas JM, Nguyen H, Li T, et al. Hormone-dependent regulation of BRCA1 in human breast cancer cells. Cance Res 1995;55:4561-5.
6 Fan S, Wang J-A, Yuan R, et al. BRCA1 inhibition of estrogen receptor signaling in transfected cells. Science 1999; gen receptor

7 Koonin EV, Altschul SF, Bork P. BRCA1 protein products. Functional motifs. Nat Genet 1996;13:266-8.

8 Chapman MS, Verma IM. Transcriptional activation by BRCA1. Nature 1996;382:678-9.

9 Monteiro AN, August A, Hanafusa H. Evidence for a transcriptional activation function of BRCA1 C-terminal region. Proc Natl Acad Sci US A 1996;93:13595-9.

10 Kote-Jarai Z, Eeles RA. BRCA1, BRCA2 and their possible function in DNA damage response. Br f Cancer 1999;81: 1099-102.

11 Gowen LC, Avrutskaya AV, Latour AM, et al. BRCA1 required for transcription-coupled repair of oxidative DNA damage. Science 1998;281:1009-12.

12 Shao N, Chai YL, Shyam E, et al. Induction of apoptosis by the tumor suppressor protein BRCA1. Oncogene 1996;13: the tum.

13 Cortez D, Wang Y, Qin J, et al. Requirement of ATM-dependent phosphorylation of BRCA1 in the DNA damage response to double-strand breaks. Science 1999; 286:1162-6.

14 Chen Y, Chen C-F, Riley DJ, et al. Aberrant subcellular localization of BRCA1 in breast cancer. Science 1995;270: 789-91.

15 Scully R, Ganesan S, Brown M, et al. Location of BRCA1 in human breast and ovarian cancer cells. Science 1996;272: $123-5$.

16 Jensen RA, Thompson ME, Jetton TL, et al. BRCA1 is secreted and exhibits properties of a granin. Nat Genet 1996;12:303-8

17 Coene E, Van-Oostveldt P, Willems $\mathrm{K}$, et al. BRCA1 is localized in cytoplasmic tube-like invaginations in the nucleus. Nat Genet 1997;16:122-4.

18 Wilson CA, Ramos L, Villaseñor MR, et al. Localization of Wilson CA, Ramos L, Villasenor MR, et al. Localization of
human BRCA1 and its loss in high-grade, non-inherited breast carcinomas. Nat Genet 1999;21:236-40.

19 Yoshikawa K, Honda K, Inamoto T, et al. Reduction of BRCA1 protein expression in Japanese sporadic breast carcinomas and its frequent loss in BRCA1-associated cases. Clin Cancer Res 1999;5:1249-61.

20 Taylor J, Lymboura M, Pace PE, et al. An important role for BRCA1 in breast cancer progression is indicated by its loss in a large proportion of non-familial breast cancers. Int $\mathcal{f}$ Cancer 1998;79:334-42.

21 Bièche I, Noguès C, Lidereau R. Overexpression of BRCA2 gene in sporadic breast tumours. Oncogene 1999:18:5232-8.

2 Gonzalez R, Silva TM, Dominguez G, et al.Detection of heterozygosity at RAD51, RAD 52, RAD 54 and BRCA1 and BRCA2 loci in breast cancer: pathological correlations. Br f Cancer 1999;81:503-9.

23 Hartmann LC, Schaid DJ, Woods JE, et al. Efficacy of bilateral prophylactic mastectomy in women with a family history of breast cancer. N Engl f Med 1999;340:77-84.

24 Azzopardi JG, Chepick OF, Hartmann WH, et al. The World Health Organisation histological typing of breast tumors - second edition. Am f Clin Pathol 1982;78:113-23.

25 Frierson HF, Wolber RA, Berean KW, et al. Interobserver reproducibility of the Nottingham modification of the Bloom and Richardson histologic grading scheme for infiltrating ductal carcinoma. Am f Clin Pathol 1995;103:195-8.

26 Jarvis EM, Kirk JA, Clarke CL. Loss of nuclear BRCA1 expression in breast cancers is associated with a highly proliferative tumor phenotype. Cancer Genet Cytogenet 1998; 101:109-15.

27 Wilson CA, Payton MN, Pekar SK, et al. BRCA1 protein products: antibody specificity. Nat Genet 1996;13:264-5.

28 Bernard-Gallon DJ, Crespin NC, Maurizis JC, et al. Crossreaction between antibodies raised against the last 20 C-terminal amino acids of BRCA1 $(\mathrm{C}-20)$ and human EGF and EGF-R in MCF 10a human mammary epithelial cell line. Int f Cancer 1997;71:123-6.

29 Bernard-Gallon DJ, Peffault de Latour M, Rio PG, et al. Subcellular localization of BRCA1 protein in sporadic breast carcinoma with or without allelic loss of BRCA1 gene. Int F Oncol 1999;14:653-61.

30 Wilson CA, Payton MN, Elliott GS, et al. Differential subcellular localization, expression and biological toxicity of BRCA1 and the splice variant BRCA1- $\Delta 11 \mathrm{~b}$. Oncogene 1997;14:1-16.

31 Kainu T, Kononen J, Johansson O, et al. Detection of germline BRCA1 mutations in breast cancer patients by quantitative messenger RNA in situ hybridization. Cancer Res 1996; 56:2912-15.

32 Thakur S, Zhang HB, Peng Y, et al. Localization of BRCA1 and a splice variant identifies the nuclear localization and a splice variant identifies the
signal. Mol Cell Biol 1997;17:444-52.

33 Ribieras S, Magdinier F, Leclerc D, et al. Abundance of BRCA1 transcripts in human cancer and lymphoblastoid cell lines carrying BRCA1 germ-line alterations. Int $\mathcal{F}$ Cancer 1997;73:715-18

34 Lee W-Y, Jin Y-T, Chang T-W, et al. Immunolocalization of BRCA1 protein in normal breast tissue and sporadic invasive ductal carcinomas: a correlation with other biological parameters. Histopathology 1999;34:106-12.

35 Thompson ME, Jensen RA, Obermiller PS, et al. Decreased expression of BRCA1 accelerates growth and is often present during sporadic breast cancer progression. Nat Genet 1995;9:444-50.

36 Rio PG, Maurizis J-C, Peffault de Latour M, et al. Quantification of BRCA1 protein in sporadic breast carcinoma with Cancer 1999;80:823-6. 